Bernard POUDERON

(Université de Tours)

\title{
NOUVEAU TÉMOIGNAGE SUR L'ORIGINE ORPHICO-PYTHAGORICIENNE DE LA MÉTAPHORE D'ULYSSE COMME SYMBOLE DE L'ÂME (A propos du récent ouvrage de Madeleine Scopello)
}

Dans un des articles réunis au sein du bel ouvrage publié tout récemment par Madeleine Scopello figure une étude sur l'Exégèse de l'âme, dans laquelle elle soutient l'origine judéo-hellénistique des citations poétiques insérées au sein de ce véritable „petit roman” de l'âme ${ }^{1}$. J'ai déjà publié il y aura bientôt trois ans une étude portant sur l'origine du mythe d'Ulysse et d'Hélène comme deux symboles de l'âme humaine ${ }^{2}$, dans laquelle je m'efforçais de montrer que les citations d'Homère qu'utilisait le rédacteur anonyme de l'Exégèse prenaient tout leur sens si on les rattachait à un milieu médio-platonicien ou orphicopythagoricien, et que dès lors il n'y avait guère de raison de supposer une origine judéo-hellénistique au recueil de testimonia poétiques sur l'âme utilisé dans l'Exégèse.

La faiblesse relative de ma démarche résidait sans doute dans le fait que je faisais appel d'une part à une représentation figurée, le fameux bas-relief de la „basilique” orphico-pythagoricienne de la Porte Majeure à Rome, dont l'interprétation peut toujours prêter à contestation, et d'autre part à des témoignages relativement tardifs, trop peut-être pour que je puisse étayer avec suffisamment de force mon hypothèse que, déjà, au $\mathrm{I}^{\mathrm{er}}$ siècle de notre ère, le personnage d'Ulysse était régulièrement utilisé pour symboliser l'âme dans une perspective eschatologique, et non simplement psychologique ou morale. Le hasard de mes lectures, mais aussi l'utilisation du Thesaurus numérisé de l'Université d'Irvine m'ont permis de découvrir un texte qui conforte ma position. Mais rappelons d'abord à notre public la problématique et l'enjeu de ce débat.

${ }^{1}$ Cfr. M. Scopello, Femme, gnose et manichéisme. De l'espace mythique au territoire du réel (Nag Hammadi \& Manichean Studies n53), Leiden 2005, 192, renvoyant à ses deux études: Les testimonia dans le traité de l'Exégèse de l'âme, RHR 191-192 (1977) 159-171, et Les citations d'Homère dans le traité de l'Exégèse de l'âme, dans: Gnosis and Gnosticism, éd. M. Krause, Leiden 1977, 3-12.

${ }^{2}$ Cfr. B. Pouderon, Hélène et Ulysse comme deux âmes en peine: une symbolique gnostique ou pythagoricienne?, REG 116 (2003) 132-151. 
L'Exégèse de l'âme (NHC II, 6) semble représenter la version primitive du mythe gnostique de Sophia-Ennoia, bien connu par la version qu'en donne Irénée dans son Adversus haereses ${ }^{3}$. Dans ce premier état du mythe, Psychè, l'âme, est présentée comme une jeune vierge qui abandonne la maison de son père, c'est-à-dire le monde céleste, pour descendre parmi les hommes et y prendre corps. Cette „katabase” est pour elle l'occasion de terribles épreuves; elle connaît même la déchéance, puisque ses différents amants abusent d'elle et la prostituent. Elle regrette donc amèrement sa faute, implore son père, qui lui accorde son repentir; l'âme „convertie" rejoindra donc finalement la patrie céleste d'où elle est issue ${ }^{4}$.

Les lamentations de l'âme ressortissent à deux univers allégoriques: d'une part celui de l'Isral prostitué, dans des versets empruntés aux livres de Jérémie, d'Osée et d'Ezéchiel ${ }^{5}$, et d'autre part celui de la „nostalgie” d'Hélène et d'Ulysse, exprimant l'un et l'autre dans l'Odyssée le désir de retrouver au plus vite leur foyer et leur patrie ${ }^{6}$. Seul nous intéresse ici le personnage d'Ulysse. Voici le passage où il apparaît dans l'Exégèse, sous la forme de brefs renvois à deux (au moins) passages distincts de l'Odyssée.

\section{Exégèse de l'âme}

p. 136, 27-35: C'est pourquoi il est écrit dans le poète qu'Ulysse était assis sur l'île, pleurant et triste, détournant son visage des paroles de Calypso et de ses tromperies, désirant voir son village et une fumée qui s'en élève.

\section{Parallèles dans l'Odyssée}

I, 48-50, et 55-59: J'ai le cœur brisé pour Ulysse, pour ce sage, accablé du sort, qui, loin des siens, continue de souffrir dans une île aux deux rives; la fille d'Atlas tient captif le malheureux qui pleure, $[\ldots]$ ne voulant que voir monter un jour les fumées de sa terre; $\mathbf{I V}, \mathbf{5 5 5 - 5 5 8}$ : Je l'ai vu dans une île pleurer à chaudes larmes; là-bas, dans son manoir, Calypso, de force, le retient.

Le rédacteur établit donc un parallèle entre les errances d'Ulysse loin de sa terre natale, qui symbolise le monde céleste, et celles de la malheureuse Psychè, tombée d'en haut jusque dans l'univers matériel. Seul le rapport que Madeleine Scopello établit avec la première séquence de citations, celle qui puise dans les textes prophétiques évoquant la prostitution d'Isral, permet de supposer que l'une et l'autre séries proviennent d'une ou plusieurs collections

\footnotetext{
${ }^{3}$ Cfr. Irenaeus, Adversus haereses I 2, 1-2.
}

${ }^{4}$ Cfr. J.M. Sévrin (éd), L'Exégèse de l'âme, BCNH nº 9, Québec 1983; M. Scopello (éd), L'Exégèse de l'âme, NHS n²5, Leiden 1985.

${ }^{5}$ Cfr. Exegesis animae p. 129, 5-131, 13: citations de Jr 3, 1-4; Os 2, 4-9; Ez 16, 23-26a; agraphon: 1 Co 5, 9-10; voir Scopello, Les testimonia, cité à la note 1 .

${ }^{6}$ Cfr. Exegesis animae p. 136, 27-137, 11; voir Scopello, Les citations, cité à la note 1; A.J. Droger, Homer Exegesis among the Gnostics, StPatr 29 (1989) 313-321. 
de textes déjà constituées et qu'elles sont issues d'un même milieu, nécessairement judéo-hellénistique.

Il importe en fait de dissocier l'origine de l'un et l'autre florilège. Le meilleur moyen de le faire est de montrer qu'il existait dans le monde grec, en dehors de toute influence juive ou chrétienne, une tradition bien établie identifiant les errances d'Ulysse à celle de l'âme sur le chemin qui la ramène vers son lieu d'origine. Je livrerai d'abord ici le dossier tel que je l'avais assemblé pour l'article paru dans la Revue des Études Grecques auquel j'ai déjà fait référence, avant d'y ajouter une nouvelle pièce, particulièrement intéressante à mes yeux.

\section{LE DOSSIER EXISTANT}

Pour qu'on puisse soutenir qu'il existait déjà au $\mathrm{I}^{\mathrm{er}}$ siècle, en milieu païen, une ou plusieurs collections de textes poétiques, et plus spécialement de vers homériques, évoquant le devenir de l'âme sous forme allégorique en utilisant soit le personnage d'Ulysse, soit des épisodes proprement odysséens, il importait de trouver en nombre suffisant des textes qui utilisassent cette matière, soit par des citations directes d'Homère, soit par de simples allusions à ses poèmes. D'autre part, il convenait aussi de distinguer les différents emplois de ces citations: le compilateur les utilisait-il dans le même contexte que le rédacteur de l'Exégèse, c'est-à-dire dans une perspective uniquement eschatologique, ou au contraire dans un cadre plus largement psychologique, pour désigner des tendances, des forces ou des parties de l'âme? Enfin, leur datation était déterminante; en effet, on ne peut tirer aucun indice suffisamment probant d'un usage qui se serait fait à des époques où la „culture” chrétienne s'était largement développée, et atteignait même les couches païennes.

Voici donc les parallèles que nous avons établis, en partie à partir des travaux de nos prédécesseurs, et en partie grâce à nos propres recherches. Nous les classons en deux groupes, d'une valeur démonstrative inégale, selon que l'allégorie porte très généralement sur l'âme, ou plus particulièrement sur le cycle de l'incarnation.

\section{Ulysse comme symbole de l'âme en tant qu'élément supérieur du} composé humain. Le plus ancien témoignage de l'usage allégorique du personnage d'Ulysse pour désigner un type humain, un trait de caractère ou un mode de vie, est celui de Maxime de Tyr (un contemporain de Commode selon la $\left.\operatorname{Souda}^{7}\right)$ :

${ }^{7}$ Cfr. Suidae lexicon, éd. A. Adler, III, s.v. Máguos, p. 321, nº 173; voir Der neue Pauly, VII, Stuttgart - Weimar 1999, 1074-1075. 
„Pour Homère aussi Ulysse, dans sa longue errance qui a fait de lui un sage, «a vu bien des cités des hommes et connu leur esprit» (Odyssea I 3) [...]. Son âme s'élève au-dessus de la terre jusqu'au ciel, [...] elle s'unit au chœur des astres [...] et peu s'en faut qu'elle n'administre l'univers et règle sa marche aux côtés de Zeus",

On constatera que la perspective n'est pas tant eschatologique que psychologique et cosmique: il s'agit ni plus ni moins que d'un éloge du sage, dont Ulysse est le modèle son âme, qui a su se dégager des pesanteurs terrestres, est immortalisée par avance, dans une vision très pythagorisante (ou stoïcisante? $)^{9}$, sous la forme d'un astre; c'est une allusion à ce qu'on désigne généralement par les termes d'immortalité astrale, dont le Songe de Scipion, à la fin de la République de Cicéron ${ }^{10}$, nous fournit un excellent exemple. Et Plotin $(205-270)^{11}$ ne dit rien d'autre, quand il donne ce conseil à ses disciples:

\begin{abstract}
„Enfuyons-nous dans notre chère patrie (i.e. le monde incorporel), voilà le vrai conseil qu'on pourrait nous donner. Mais qu'est cette fuite? Comment remonter? Comme Ulysse, qui échappa, dit-on, à Circé la magicienne et à Calypso, c'est-àdire qui ne consentit pas à rester près d'elles, malgré les plaisirs des yeux et toutes les beautés sensibles qu'il y trouvait" ${ }^{\text {, }}$.
\end{abstract}

${ }^{8}$ Maximus Tyrensis, Dissertationes 16, 6, éd. G.L. Koniaris, Berlin - New York 1995, 206-207; le discours porte le titre suivant: „Que la vie théorétique est meilleure que la vie pratique”.

${ }^{9}$ L'immortalité astrale peut de fait être rattachée au pythagorisme; cfr. Macrobius, Commentarius in somnium Scipionis I 12, 3, éd. J. Willis, Leipzig 1994: „C'est ce que symbolise la divine sagesse d'Homère, dans sa description de l'antre d'Itaque. C'est aussi ce qui fait penser à Pythagore que l'empire de Dis commence au-dessous de la Voie lactée; car on voit bien qu'une fois qu'elles l'ont quittée, les âmes ont rompu avec les régions supérieures..."; voir aussi Héraclide le Pontique, chez Stobée (Anthologium I 378, 12, éd. C. Wachsmuth - O. Hense, Berlin 1958); Manilius, Astronomica I 758-761, éd. G.P. Goold, Cambridge 1997: „Est-ce que les âmes courageuses et les personnes dignes du ciel, une fois débarrassées de leur corps et restituées à la terre, migrent de l'orbe terrestre jusque là, et, habitant un ciel devenu leur, vivent des années éthérées et jouissent de leur (séjour) cosmique?"; mais on peut aussi penser à la doctrine du médio-stö̈cien Posidonius, sur lequel s'exerça l'influence de Pythagore: F 151, éd. L. Edelstein - I.G. Kidd, New York $1988=$ Galenus, De placitis Hippocratis et Platonis éd. C.G. Kühn, V, Leipzig 1884, 478.

${ }^{10}$ Cfr. Cicero, Respublica chez Macrobe (Commentarius in somnium Scipionis II 17, 1-2, éd. M. Armisen-Marchetti, Paris 2001, p. 83): „Donc, après son exposé didactique sur le mouvement de l'âme, l'Africain décrit et prescrit en ces termes la façon d'en faire usage: «Exerce-la aux entreprises éminentes; or éminents sont les soins que l'on donne au salut de la patrie; l’âme qui s'y sera adonnée et exercée prendra plus vite son envol vers ce séjour et cette demeure qui sont les siens; et elle le fera d'autant plus rapidement que, alors même qu'elle sera encore enfermée dans le corps, elle s'élèvera au dehors et, par la contemplation du monde extérieur, s'arrachera le plus possible au corps. Car pour ceux qui se sont abandonnés aux plaisirs physiques, qui se sont mis en somme à leur service, et qui, sous l'impulsion des désirs dociles aux plaisirs, ont violé le droit divin et humain, leurs âmes, une fois qu'elles ont échappé au corps, tournent autour de la terre, et ne retrouvent le lieu où nous sommes qu'après bien des siècles de tourments»".

11 Cfr. Der neue Pauly, IX 1146-1155.

12 Plotinus, Enneades I 6, 8; cfr. F. Buffière, Les mythes d'Homère et la pensée grecque, Paris 1956, 417; J. Pépin, Mythe et allégorie, Paris 1958, 199-200. 
Le témoignage de Porphyre (234-305/310) ${ }^{13}$, trop tardif - il est sans doute postérieur à la rédaction de l'Exégèse de l'âme ${ }^{14}$, et a fortiori à l'élaboration d'un éventuel recueil de citations auquel son rédacteur aurait puisé -, est en revanche beaucoup plus proche par l'esprit du contexte de l'Exégèse sur l'âme:

„Et ainsi Ulysse eut du mal à quitter Calypso, en tant qu'il était naturellement attaché à la vie. Mais, par l'intermédiaire d'Hermès, c'est-à-dire de la raison, il a retrouvé sa patrie philosophique tant désirée, c'est-à-dire le monde philosophique, vraie patrie des âmes, aux yeux des Platoniciens" ${ }^{\text {15. }}$.

Il s'agit non seulement de voir dans Ulysse un symbole de l'âme, mais encore de voir dans ses povnoi le symbole des efforts du sage pour parvenir jusqu'au monde des intelligibles, ce que Porphyre appelle le „monde philosophique”. La perspective eschatologique, même si elle reste envisageable, ne me semble pas dominer.

Bien plus tard encore, le néoplatonicien Proclus $(412-485)^{16}$ prolongera l'exégèse porphyrienne en voyant dans le retour d'Ulysse sur son île le repos de l'âme propre à ceux qui vivent selon l'esprit:

„Seule la vie selon l'esprit procure cette fixité et ce mouillage mystique de l'âme où le poète conduit Ulysse, après la grande errance de la vie" ${ }^{17}$.

Mais aucun des ces trois intellectuels ne fait des errances d'Ulysse le symbole du voyage de l'âme incarnée sur le chemin du retour vers sa patrie céleste, ni d'Ulysse lui-même le symbole de l'âme immortelle prisonnière d'un corps mortel.

\section{Ulysse comme symbole de l’âme en tant qu'élément immortel empri-} sonné dans un corps mortel. Cette thématique, en revanche, nous la trouvons,

${ }^{13}$ Cfr. Der neue Pauly, X 174-181.

${ }^{14}$ Cfr. Sévrin, op. cit., p. 59-60: terminus post quem: c. 120 (diffusion avérée de l'évangile de Jean); terminus ante quem: c. 135 (départ de Valentin pour Rome; début de la diffusion de sa doctrine, apparemment inconnue du rédacteur de l'Exégèse).

15 Porphyrius, Antrum Nympharum 46-50, cité par Buffière, op. cit., p. 463, note 1. Parall. Eustathius, Commentarii ad Homeri Odysseam I 1389, 46-49, éd. G. Stallbaum, Leipzig 1825, p. 17: „C'est par l'intermédiaire d'Hermès, comme l'indiquera plus loin le poète, c'est-à-dire de la raison, qu'Ulysse a retrouvé sa patrie philosophique qu'il désirait tellement, soit le monde intelligible. Ce qui est, selon les platoniciens, la vraie patrie des âmes. Tout de même il a retrouvé Pénélope, la philosophie, après avoir été délié et débarrassé de cette Calypso" (cité par M.-H. Quet, Naissance d'image: la mosaique des Thérapénides d'Apamée de Syrie, représentation figurée des connaissances encycliques, servantes de la philosophie hellène, „Cahiers du Centre Gustave Glotz” 4:1993, 129-187, ici p. 137, note 41).

${ }^{16}$ Cfr. Der neue Pauly, X 383-388.

17 Proclus, In Platonis Parmenidem 1, 1025, 32; cité par Buffière, op. cit., p. 418. 
plus ou moins explicitée, chez d'autres philosophes. Le premier d'entre eux est Nouménios d'Apamée ${ }^{18}$, un médio-platonicien fortement teinté de néo-pythagorisme, qui a vécu dans la seconde moitié du $\mathrm{II}^{\mathrm{e}}$ siècle, comme l'attesterait la première mention qui est faite de lui dans la littérature, à savoir chez Clément d'Alexandrie. Le fragment qui fait allusion aux errances de l'âme est conservé chez Porphyre, qui nous apprend par ailleurs que Nouménios était lu au sein de l'école de Plotin ${ }^{19}$ :

„À mon avis, Nouménios et ses disciples croyaient non sans raison qu'Ulysse, dans l'Odyssée d'Homère, figurait celui qui passe par les cycles successifs de la génération ( ceux qui sont à l'abri de toute vague et de toute mer"20.

À peine moins explicite est le témoignage du néoplatonicien Hermias

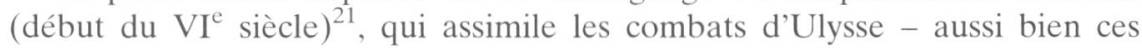
exploits guerriers dans l'Iliade que ses errements sur le chemin du retour dans l'Odyssée - au destin de l'âme, désireuse d'échapper à la matière:

„Les exégètes les plus profonds de l'Iliade et de l'Odyssée « évoquent à ce propos aussi la remontée «de l'âme» [...]; c'est pourquoi, affirment-ils, puisque l'âme bataille pour sortir de la matière, «le poète» a représenté des combats, des batailles et autres (affrontements), tandis que, dans l'Odyssée, «il représente Ulysse» naviguant dans les parages des Sirènes, échappant à Circé, aux Cyclopes, à Calypso et à tout ce qui fait obstacle à l'élévation de l'âme et, après cela, à son retour dans sa patrie, c'est-à-dire l'intelligible"22.

Dans ce dernier texte, nous semble-t-il, se mêlent deux niveaux d'interprétation: l'un assimilant les voyages d'Ulysse à l'élévation spirituelle de l'âme dans sa quête de l'absolu, l'autre à son retour dans son milieu d'origine après sa séparation d'avec le corps. Cette ambiguité n'est pas surprenante en soi; on trouve la même ambivalence dans l'approche gnostique de la résurrection, conçue tantôt comme un phénomène intérieur, une „palingénésie”, une rénovation de son être profond, et tantôt comme une espérance par delà la mort, à l'instar de la résurrection corporelle prêchée au sein de la grande Église.

18 Cfr. Der neue Pauly, XVIII 1050-1052.

19 Cfr. Porphyrius, Vita Plotini 14, 10-12.

${ }^{20}$ Numenius, chez Porphyre (Antrum nympharum 34); voir Buffière, op. cit., p. 414.

${ }^{21}$ Cfr. P. Maraval, Hermias de Phénicie, dans: Dictionnaire des philosophes antiques, dir. R. Goulet, III, Paris, 2000, p. 651-652, nº 81.

22 Hermias, In Platonis Phaedrum scholia, éd. P. Couvrer, p. 214 (= p. 367 Bernard) - un passage, jusque là, passé inaperçu, malgré son importance. Hermias d'Alexandrie est un philosophe néoplatonicien contemporain (et condisciple) de Proclus; voir l'introduction de la traduction de H. Bernard: Hermeias von Alexandrien, Kommentar zu Platons „Phaidros”, Tübingen 1997. 


\section{UN NOUVEAU TEMOIGNAGE}

Est-il possible que Nouménios, en assimilant les errances d'Ulysse à la destinée de l'âme, n'ait fait que suivre une tradition juive, puisque nous savons par ailleurs que le philosophe d'Apamée éprouvait une réelle sympathie pour le monothéisme juif, qu'il comparait volontiers à la doctrine de Platon ${ }^{23}$ - une tradition que l'on retrouverait dans la source de l'Exégèse sur l'âme? Si l'on suit le raisonnement de Madeleine Scopello, c'est l'hypothèse à laquelle on aboutirait nécessairement.

C'est tout l'intérêt du nouveau témoignage que j'ai recueilli, que de montrer que même l'exégèse eschatologique des errances d'Ulysse appartient bel et bien à la tradition du médio-platonisme et qu'elle n'est pas une création judéohellénistique. En effet, on en trouve une excellente illustration dans un fragment de Plutarque conservé chez Stobée, le fragment $178^{24}$. En voici d'abord le contexte, puis le texte, dans ma propre traduction.

1. Le contexte. Plutarque, par la bouche de son personnage Timon, présente la mort comme une initiation (aux mystères), rapprochant le verbe $\tau \varepsilon \lambda \varepsilon v \tau \tilde{\alpha} v$, „mourir”, de $\tau \varepsilon \lambda \varepsilon \tilde{\tau} \sigma \theta \alpha$, ,être initié”, et il en décrit les étapes: d'abord les errances ( $\pi \lambda \alpha \dot{v} \alpha \iota)$ dans l'obscurité, puis la peur (

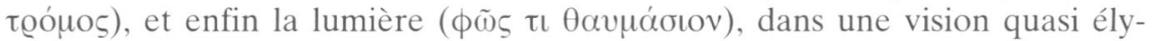

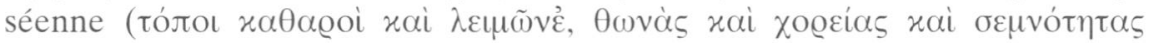

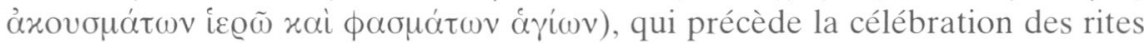
de l'initiation, destinée à distinguer le myste de la foule des non-initiés, mar-

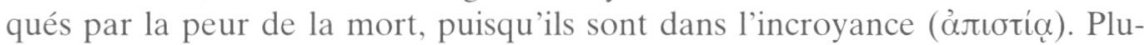
tarque poursuit en jugeant „,contre nature” le lien ( $\sigma v \mu \tau \lambda \mathrm{o} x \eta \eta$ ) de l'âme et du corps et l'emprisonnement ( $\sigma u ́ v \varepsilon \varrho \xi ı$ ) de l'une dans l'autre. Suit un développement sur le sommeil, qui nous délivre de notre condition soumise à la

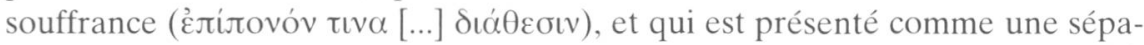
ration provisoire de l'âme et du corps, comme la mort en est une définitive (iò

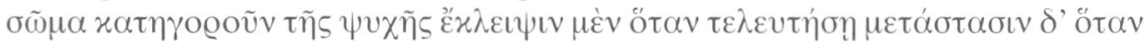
$x \alpha \theta \varepsilon v ́ \delta \eta\rceil)$. Puis Timon, en réponse à une objection de son interlocuteur, Patrocléas, explique pourquoi le réveil, dans la vie ordinaire, n'est pas une douleur: c'est que l'âme s'est habituée à sa condition de veille. C'est là que se situe notre passage sur Ulysse; le fragment le plus intéressant est celui qui assimile les efforts d'Ulysse pour échapper à la mortifère Charybde en se cramponnant de toutes ses forces à un figuier, à la lutte de l'âme qui, si elle n'est pas initiée aux mystères de la mort, s'accroche désespérément au corps qui l'emprisonne, par peur de l'inconnu.

${ }^{23}$ D'après Clément (Stromata I 22, 150,4) = Numenius, Fragmenta et testimonia 8, éd. É. des Places.

${ }^{24}$ Plutarque vécut de 45 à 125 au plus tard, cfr. Der neue Pauly, IX 1159-1176. 


\section{Le texte. En voici le texte:}

De la même façon (pour) l'âme: du fait de la communauté (qui la lie) aux passions, aux membres et aux organes mortels, ce qui est contre nature et étranger à elle ne semble pas l'oppresser outre mesure, suite à une longue habitude; mais elle ressent un soulagement et un allègement accompagné de plaisir quand elle se détache de ses activités corporelles; car ce sont elles qui la troublent, ce sont elles qui l'épuisent, c'est loin d'elles qu'elle a besoin de tranquillité et de repos.

Car pour ce qui est des activités qui sont les siennes propres, c'est-à-dire le fait d'examiner toujours quelque objet, d'y réfléchir, de le garder en mémoire et de le contempler, elle y est infatigable et insatiable. En effet, la satiété semble être une lassitude dans les plaisirs due au fait que l'âme éprouve quelque (sentiment douloureux) (quand elle est associée) avec le corps, puisque, pour ce qui est de ses propres plaisirs, elle ne fléchit pas; mais enlacée, comme on l'a dit, au corps, elle connaît les mêmes (sentiments) qu'Ulysse;

en effet, de même que lui, il se tenait fixé au figuier et qu'il l'embrassait sans le désirer ni l'aimer, mais parce qu'il redoutait la présence de Charybde (Odyssea XII 432), de même l'âme semble tenir «au corps" et l'enlacer sans (éprouver) aucune bienveillance ni reconnaissance envers lui, mais par horreur de l'inconnu de la mort.

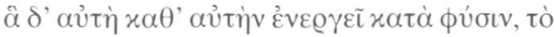

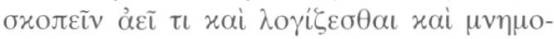

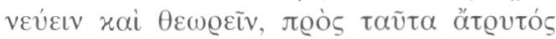

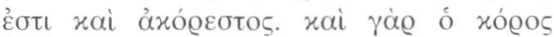

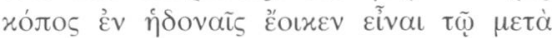

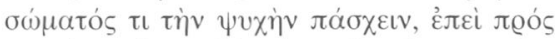

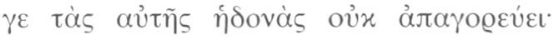

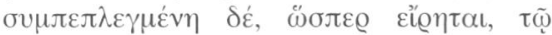

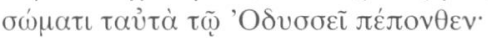

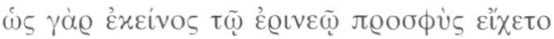

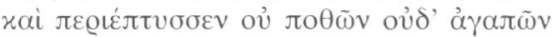

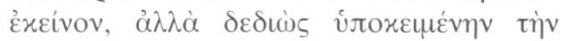

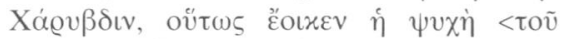

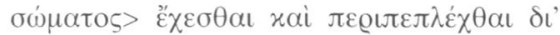

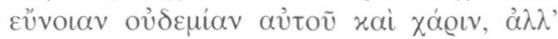

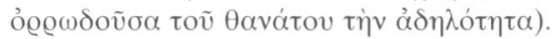

C'est que les dieux ont caché aux hommes ce qui les fait vivre (Opera et dies 42), s'il faut en croire le sage Hésiode, en n'attachant pas étroitement l'âme au corps par des liens charnels, mais en imaginant pour elle un seul lien, une seule prison pour l'envelopper, l'inconnaissance et l'incertitude de ce qui se passe après la mort; car (l'âme) qui serait confiante en ce qui attend les hommes après leur mort, selon Héraclite, rien ne saurait la retenir (frag. 27 D.-K.).

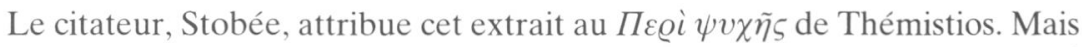
une telle attribution est absolument impossible, puisque, au début du III $^{\mathrm{e}}$ siècle, Clément cite déjà plusieurs fragments du passage, sans indication d'auteur, il est vrai. L'attribution à Plutarque est le fait de M.R. James, dans un article de la $C R$ de $1900^{25}$. Elle repose sur l'existence d'un $\Pi \varepsilon \varrho i$ $\psi v \chi \tilde{\eta} \varsigma$ attribué à Plutarque, existence attestée par les mentions et citations qu'en font Origène, Aulu-Gelle et Eusèbe; sur l'apparition des personnages de Timon, le frère de Plutarque, et Patrokléas, qui sont les interlocuteurs de notre Пع@i $\psi v \chi \tilde{\eta} \varsigma$, dans deux autres ouvrage de Plutarque, le De sera numinis vindicta

25 Cfr. M.R. James, Clement of Alexandria and Plutarch, ,The Classical Review” 14 (1900) $23-24$ 
(548a-568a), et les Quaestiones convivales (612c-748d); sur la parenté de style qui unit ce fragment à d'autres passages de Plutarque; et encore sur la datation la plus probable, à savoir le $\mathrm{I}^{\mathrm{er}}$ siècle de notre ère ou la première moitié du second, compte tenu de l'usage que fait Clément du texte.

Quel que soit son auteur, le passage peut de toute façon être daté avec certitude comme étant au plus tard de la fin du $\mathrm{II}^{\mathrm{e}}$ siècle. Il ne témoigne d'aucune influence juive, mais bien au contraire puise dans les deux traditions platonicienne et orphico-pythagoricienne. Les principaux éléments platoniciens sont la croyance en l'immortalité de l'âme, présentée comme formant l'essence véritable de l'individu, le corps n'en étant que le véhicule

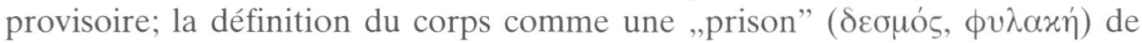
l'âme; et la caractérisation des activités de l'âme comme étant d'essence

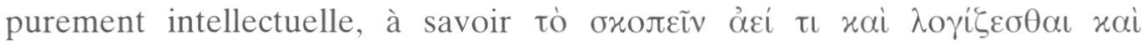

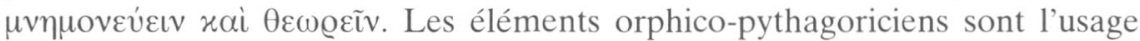

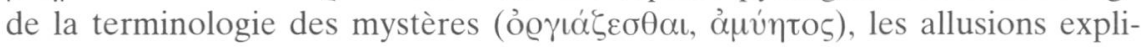
cites au rituel éleusinien, dont on sait combien son évolution a été influencée par l'orphisme $e^{26}$, et, plus généralement, la comparaison de la mort à une initiation (avec le rapprochement entre $\tau \varepsilon \lambda \varepsilon v \tau \tilde{\alpha} \nu$, „mourir” et $\tau \varepsilon \lambda \varepsilon \tau \sigma \sigma \theta \alpha$, „être initié"), ou encore le thème du corps-prison de l'âme (que Platon n'a fait qu'emprunter) ${ }^{27}$.

Le fragment contient deux citations littérales d'Hésiode et Héraclite, et une allusion à l'épisode homérique de Charybde, en l'occurrence:

- Homère, Odyssea XII 432-433: Ulysse, pour ne pas être englouti par Charybde, saisit à bras le corps le grand figuier [દ̉otveò $\mu \dot{\gamma} \gamma \alpha \varsigma$ ] que portait l'écueil (Odyssea XII 103): „Je me lève sur l'eau, je saute au haut figuier, je m'y cramponne comme

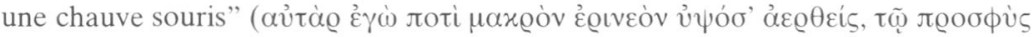

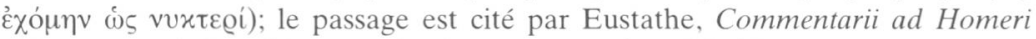
Odysseam, vol. 2, p. 34; et par Strabon, Geographica I 2, 36;

- Hésiode, Opera et dies 42: „les dieux ont caché ce qui fait vivre les hommes”

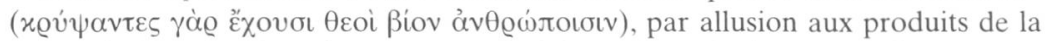
terre, plutôt qu'au feu; cité une seconde fois par Jean Stobée, Anthologium II 1, 7, ainsi que par Synésius de Cyrène, Aegyptii sive de providentia II 7, et De insomniis 1 ;

- Héraclite, frag. 27 D.-K., chez Clément, 4 Stromata IV 22, 144, 3: „les hommes doivent s'attendre, morts, à des choses qu'ils n'espèrent ni imaginent"

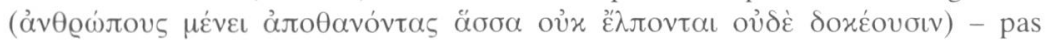
d'autre citateur connu.

${ }^{26}$ Cfr. G. Mylonas, Eleusis and the Eleusinian Mysteries, Princeton 1961, 228 et passim; W.K.C. Guthrie, Orphée et la religion grecque. Etude sur la pensée orphique, trad. fr. P. Guillemin, Paris 1956, 174-206 (à propos de la vie future), et passim.

27 Cfr. Athenaeus, Deipnosophistae IV 157c (le pythagoricien Euxithès). 
Le peu d'usage qui est fait de ces citations conduise à douter qu'elles proviennent d'une anthologie très en vogue.

En revanche, le passage de l'Odyssée contenant l'épisode d'Ulysse pleurant sur sa terre fumante (Odyssée I 48-50, et 55-59) est devenu un lieu commun de la littérature antique et moderne. Même si l'on exclut les scholies et commentaires homériques, on trouve en effet le passage cité textuellement au moins trois fois dans le monde grec tardif: chez David ${ }^{28}$ (un philosophe du VI ${ }^{\mathrm{e}}$ siècle de notre ère), qui l'a peut-être puisé chez Porphyre; chez Maxime de $\mathrm{Tyr}^{29}$, un sophiste de l'époque de Commode; et chez Ménandre le Rhéteur ${ }^{30}$, un sophiste $\mathrm{du} \mathrm{III}^{\mathrm{e}}$ siècle. Pas de citation, en revanche, chez les auteurs chrétiens (parmi lesquels je ne compte pas Eustathe, le commentateur de l'Odyssée). On opposera cette distribution à celle des testimonia monothéistes, principalement extraits de Sophocle ou Euripide (mais pas uniquement), que l'on trouve majoritairement chez des auteurs juifs et chrétiens, et dont on peut supposer qu'ils ont été, sinon entièrement forgés, du moins largement augmentés et complétés en milieu juif ${ }^{31}$.

Si l'ensemble des fragments rattachés sans conteste au Пв@i $\psi v \chi \tilde{\eta} \varsigma$ perdu de Plutarque, à savoir les fragments 173 à 176, ne fournissent aucun indice susceptible de nous guider dans notre recherche - il s'agit des fragments:

- nº173: Origenes, Contra Celsum V 57, qui mentionne les ,événements extraor-

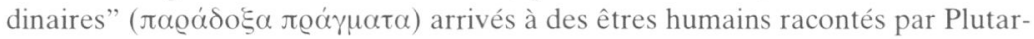
que dans son $\Pi \varepsilon \varrho \grave{~} \psi v \chi \tilde{\eta} s ;$

- n'174: Aulus Gellius, Noctes Atticae I 3, 31, avec une citation en grec du Пв@i $\psi v \chi \tilde{\eta} \varsigma$ à propos d'une sentence de Chilon l'Ancien, à qui on soutenait qu'il n'avait pas d'ennemi;

- n 175 : Aulus Gellius, Noctes Atticae XV 10, avec une allusion à un autre passage du $\Pi \varepsilon \varrho i ~ \psi v \chi \tilde{\eta} \zeta$, en fait une anecdote sur les jeunes filles de Milet, prises d'une soudaine épidémie de suicide;

- et nº176: Eusebius, Praeparatio evangelica XI 36, 1 (repris par Théodoret, Graecarum affectionum curatio XI 46), livrant, à propos du mythe d'Er le Pamphylien, une anecdote similaire concernant un dénommé Antyllos, mort puis revenu à la vie -

en revanche, le fragment 177, rattaché par erreur à Thémistios comme le

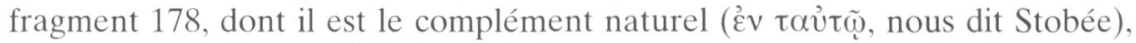
évoque de manière explicite le cycle des âmes, abandonnant la vie céleste pour

${ }^{28}$ Cfr. David, In Porphyrii Isagogen commentarium, éd. A. Busse, p. 124, 1. 26. Source: le TLG d'Irvine.

${ }^{29}$ Cfr. Maximus Sophista, Dialexeis 21, 8 c, éd. H. Hobein.

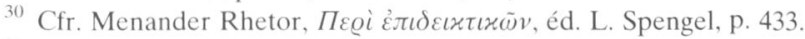

${ }^{31}$ Sur la question, cfr. N. Zeegers, Les citations des poètes grecs chez les Apologistes du second siècle, Louvain 1968. 
une prison temporaire, celle du corps et de la vie terrestre, où elle connaît les épreuves, avant de retourner goûter le repos dans le monde céleste:

- no 177: Stobaeus, Florilegia IV 52, 49, qui livre un dialogue entre Timon et Patrocléas, à propos de la croyance en l'immortalité de l'âme et de la peur de la

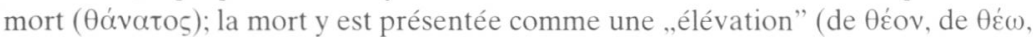

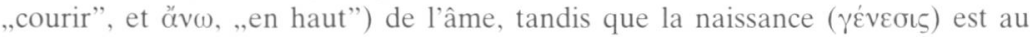

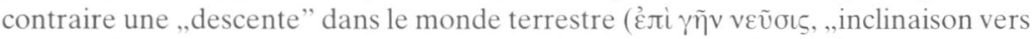

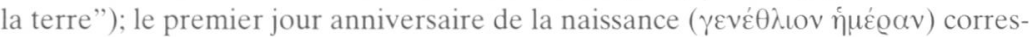

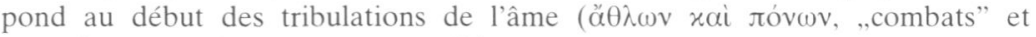

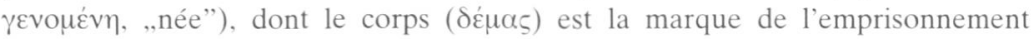
( $\delta \varepsilon \delta \varepsilon \mu \varepsilon \dot{v} \eta)$, et pour laquelle la vie terrestre (ßíos) est une violence ( $\beta i \alpha)$, selon l'usage homérique d'employer une forme thématique de préférence à une forme

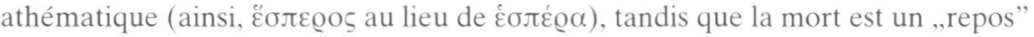

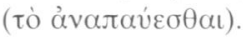

L'ambiance est homérique, comme l'indique la double référence au lan-

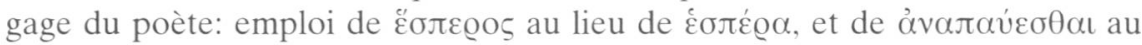

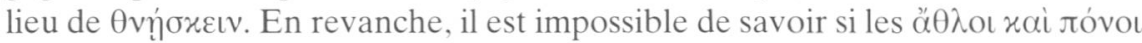
que connaît l'âme durant son séjour terrestre sont rapprochés par le moraliste plutôt des combats de l'Iliade que des épreuves subies par Ulysse dans l'Odyssée - les deux poèmes appartenant également à l'univers métaphorique des tribulations et des combats de l'âme, ainsi que l'atteste le fragment d'Hermias que nous avons cité, et, dans notre Exégèse sur l'âme, la superposition de la légende d'Hélène, personnage ,iliadique” à celui d'Ulysse, personnage ,odysséen”.

Il apparaît donc indubitable que les errances d'Ulysse appartenait déjà au $\mathrm{I}^{\mathrm{er}}$ siècle de notre ère à l'univers allégorique des tribulations de l'âme, au sein de courants liés au médio-platonisme ou au néo-pythagorisme. L'usage que fait Plutarque du personnage d'Ulysse, raccroché au figuier planté juste devant de gouffre de Charybde, comme symbole de la peur qu'éprouve l'âme à abandonner son enveloppe charnelle pour retourner dans le lieu de repos d'où elle vient, en est à mon avis une preuve suffisante. Il n'est donc pas utile de supposer que le rédacteur de l'Exégèse de l'âme a puisé dans un recueil judéo-hellénistique les citations extraites de l'Iliade et de l'Odyssée évoquant allégoriquement les voyages de l'âme tiraillée entre l'attrait du monde corporel (celui que symbolisent Pâris et Calypso) et celui du monde céleste (représenté par Sparte et Ithaque, ou bien par Ménélas et Pénélope); il est bien plus conséquent d'avancer l'hypothèse qu'il n'a fait qu'utiliser une tradition platonicienne, recourant aux figures d'Hélène et d'Ulysse, qu'il s'agisse d'un traité 
philosophique émaillé de citations homériques, comme les œuvres de Plutarque en fournissent maint exemple, ou d'un florilège de citations ad hoc, dont les aléas de la transmission textuelle ne nous ont pas conservé d'exemple constitué, mais dont la répétition incessante des mêmes citations chez les écrivains d'époque impériale, chrétiens ou païens, nous fait justement supposer l'existence. 